\title{
Bloqueo atrioventricular completo con doble interrupción del sistema conductor
}

\author{
Dr, Benedicto Chuaqui J. $\frac{1}{;}$ Dra. Odette Farrá A. 2 \\ Double intermption of the atrioventricular $(A-V)$ \\ conduction system in congenital $\mathrm{A}-\mathrm{V}$ block
}

\begin{abstract}
A case of isolated congenital A-V block in a 4 month-old girl is presented; delivery was premature, with neonatal asphyxia. Anatomopathological examination demonstrated a well developed A-V node; two fibrous interruptions of the $\mathrm{A}-\mathrm{V}$ bundle were found: one in the penetrating segment and the other in the branching segment; between them, a segment of His' bundle without specific muscular fibers and made up of only connective tissue was present. A few calcific deposits were found in the surrounding fibrous tissue. Pathogenesis of these lesions is discussed, in relation with the current ideas on the development of the conduction system in man. It is concluded that lesions found in this case do not correspond to those described in cases of A-V block due to embryonal maldevelopment, and that they are probably the result of a degenerative process affecting secondarily these structures. The possibility of neonatal hypoxia as the cause of an elective necrosis of the specific parenchyma is also considered. According to the available literature, this is the first published case of a double interruption of the $A-V$ conduction sy stem.
\end{abstract}

(Key words: Atrioventricular block, congenital interruption, conduction system).

$\mathrm{La}$ frecuencia de los bloqueos atrioventricu. lares $(\mathrm{A}-\mathrm{V})$ completos congénitos varía según los autores entre $1 \times 2.500$ partos $^{1}$ y 1 × $20.000^{2}$. En general son de buen pronóstico y rara vez conducen a la muerte en los primeros años de vida. No hemos encontrado casos publicados, en Chile, con estudio morfológico.

\section{Caso clínico}

Niña de 4 meses, nacida de parto prematuro con $2.200 \mathrm{~g}$ luego de embarazo normal. Asfixia neonatal y dificultad respiratoria transitoria. Desde el nacimiento, disnea progresiva. A los 3 meses de edad se comprobó bradicardia de 40', por lo que hospitalizó grave, con insuficiencia cardíaca, bradicardja de $40^{\prime}$ regular, soplo sistólico de eyección grado $3 / 6$, mesocardíaco; 20 ruido normal. Electrocardiograma: eje eléctrico de QRS $30^{\circ}$, bloqueo atrio-ventricular completo, frecuencia ventricular $40 \mathrm{x} \min y$ auricular $140 \mathrm{x}$ min; QRS $0,10 \mathrm{~s}$; QT prolongado

1. Departamento de Anatomía Patológica, Escuela de Medicina, Pontificia Universidad Católica de Chile.

2. Unidad de Cardiología, Departamento de Pediatría, Hospital Roberto del Río.
$(0,45 \mathrm{~s}$.); acentuados signos de reacción ventricular izquierda. Radiografía de tórax: hiperemia pulmonat pasiva y agrandamiento del ventrículo izquierdo.

Se trató con metaproterenol, $5 \mathrm{mg}$ oral cada 6 horas, sin respuesta. Al cuarto día la frecuencia había bajado a $33 \mathrm{x}$ min con frecuentes extrasístoles, por lo que se colocó marcapaso, modelo Xytron-RA 5955, de demanda, con una frecuencia de $100 \times \min$ y electrodo epimiocárdico modelo 6917-35 A. A los 21 días fue dada de alta en buenas condiciones, sin insuficiencia cardiaca congestiva. Dos días después de alta, tuvo tos, dificultad respiratoria y fiebre, motivo por el cual reingresó en grave estado, con marcada disnea, y falleció pocas horas después.

En el examen patológico el corazón estaba bieu conformado, pesaba $62 \mathrm{~g}$ incluyendo callado aórtico. El pericardio tenía adherencias en relación con cables de marcapaso. En el ventrículo izquierdo había hipertrofia excéntrica de $4 \mathrm{~mm}$ de espesor. El sistema éxcito conductor (SEC) atrioventricular se examinó en cortes perpendiculares al anillo tricuspídeo en cinco bloques sucesivos que comprendían la región que va desde la desembocadura del seno coronario a la banda septal de la crista supraventricularis. Los bloques se incluyeron en parafina y se cortaron en serie. Se tiñó uno de cada cinco cortes con el método de Van Gieson. 
El nódulo A-V estaba bien configurado, (Fig. 1), en su parte superior era continuo con la musculatura auricular, bien desarrollada. El tejido nodal sin fibrosis. Ventralmente el nódulo se continuaba en su porción compacta con algunas prolongaciones que penetraban en el trigono fibroso sin atravesarlo. Faltaba la porción proximal o perforante del haz de $\mathrm{His}$ (Fig. 2). En àlgunos cortes más ventrales sólo se encontraban delgados fascículos musculares rodeados por tejido fibroso denso con depósitos de material calcáreo. En la porción membranosa el haz apareció primero adelgazado, luego amplio, con su típica configuración triangular, pero en este segmento carecía de fibras musculares $y$ se hallaba constituido sólo por tejido conectivo (Fig. 3). Más ventralmente reparecían fibras musculares en el haz, muy adelgazado en la zona de origen de las ramas izquierdas, para luego volver a desaparecer en forma de una segunda interrupción fibrosa (Fig. 4) y reaparecer como rama derecha de estructura normal; lo mismo ocurría en las ramas izqujerdas más distales.

El nódulo sinusal y el miocardio de trabajo, tenian su estructura conservada. En el pericardio se encontró inflamación crónica inespecifica y en los pulmones bronquiolitis aguda acentuada. Se

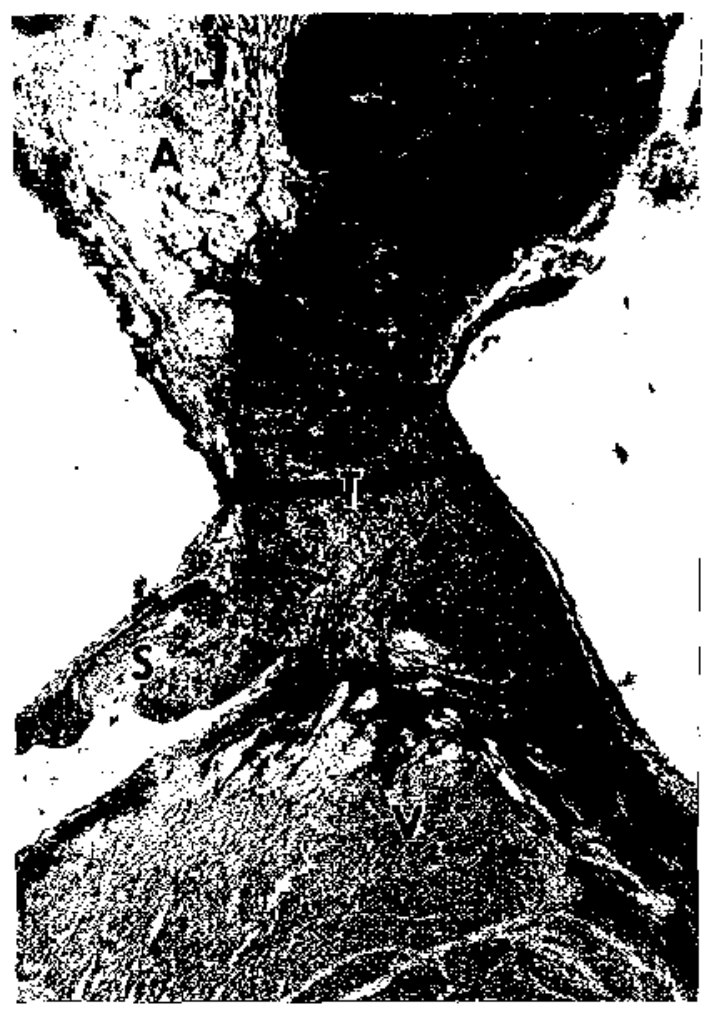

Fig. 2. Primera interrupción fibrosa del haz de His en un corte caudal a la porción membranosa. T: trígono fibroso derecho, A: musculatura del tabique interauricular, S: velo septal tricuspídeo, $V$ : tabique interventricular. Tinción de van Gieson, aumento original: $\times 32$.

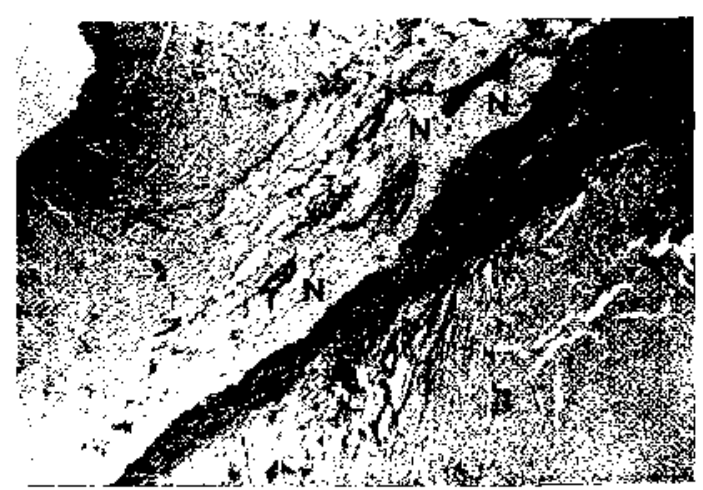

Fig. 1. Nódulo atrioventricular (N) bien conformado. Tinción de van Gieson, aumento original: $\times 32$.

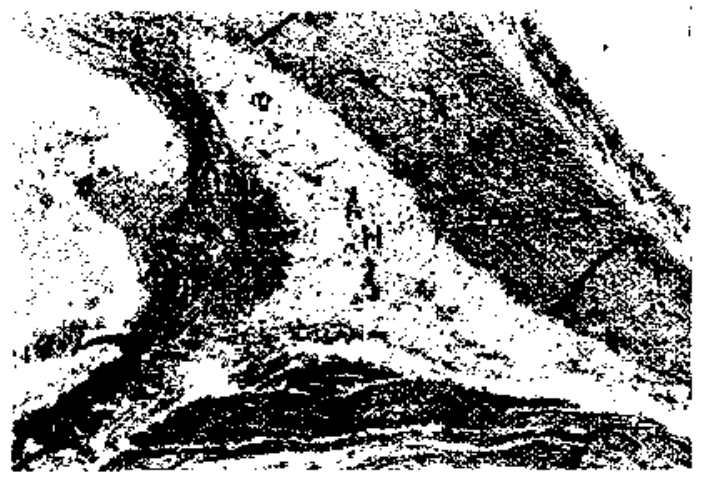

Fig. 3. Haz de $\mathrm{His}(\mathbf{H})$ en la base de la porción membranosa. El haz presesta típicamente contormos triangulares en su sección transversal, carece de musculatura específica y se halla constituido sólo por tejído conectivo. Tinción de van Gieson, aumento original: x 80 .

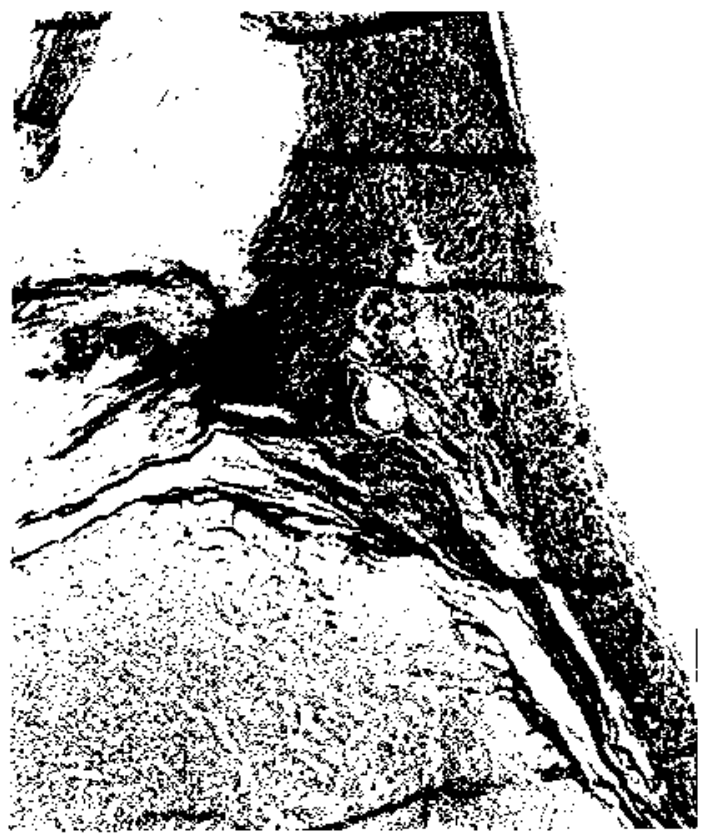

Fig. 4. Segunda interrupción fibrosa del haz de His en Ja porción ventral de la porción membranosa, en cuya base se reconocen algunos vasos y pequeños grupos aislados de fibras musculares. Tinción de van Gieson, aumento original: x 32. (Aumento adicional: aprox. x 1,5). 
hicieron los diagnósticos de interrupciones extensas del haz de His en sus porciones perforante e intramembranosa (o ramificada), con reemplazo fibroso. Ausencia de musculatura específica en el segmento restante del haz de His. Hipertrofia cardiaca ventricular izquierda excéntrica.

\section{DISCUSION}

En 1964 los mjembros de la Asociación Cardiológica Pediátrica Europea iniciaron un estudio conjunto de los bloqueos $\mathrm{A}-\mathrm{V}$ congénitos. Los resultados fueron publicados en 1967 , 1972 y 1975 y resumidos en una exhaustiva publicación de $1979^{3}$, donde se dio cuenta de más de 600 casos. En un $25 \%$ se encontraron malformaciones cardíacas asociadas, y estos casos son de peor pronóstico.

Se dice que los bloqueos aislados son de buen pronóstico, sin embargo en el período neonatal $25 \%$ tiene sintomas con $22 \%$ de letalidad; esta última es de $15 \%$ en promedio antes de los 6 meses de edad. La letalidad y morbilidad descienden en la nifhez y adolescencia $y$ vuelven a subir lentamente después de los 15 años.

En el recién nacido y lactante pequeño, la insuficiencia cardíaca y la frecuencia ventricular fija bajo $50^{\prime}$ son indicación de tratamiento, primero médico $\mathrm{y}$, si este fracasa, mediante marcapasos transitorios o permanentes.

Los factores de riesgo en el recién nacido y lactante pequeño son: frecuencia ventricular menor que $50 \mathrm{x}$ min; ocurrencia familiar; insuficiencia cardíaca; episadios de bradicardia acentuada durante el sueño (menos de $30 \times$ min); marcapasos bajo o cambiante; QT prolongado; bloqueos de rama. La frecuencia auricular alta no tiene mayor importancia a esta edad.

En nuestra paciente el bloqueo se detectó sólo en el tercer mes de vida, pero era sintomática desde el nacimiento. Es probable que al nacer haya tenido una frecuencia cardiaca mayor que luego fuera descendiendo, hecho descrito en algunos pacientes con mal pronóstico. La indicación de marcapaso fue clara: habia insuficiencia cardíaca $y$ frecuencia ventricular fija y baja que no cedió al tratamiento médico. Por la clínica y el ECG con QRS ancho y QT prolongado se podia suponer que el sitio del bloqueo era bajo, tal como se demostró anatómicamente. Cabe señalar que un QRS angosto no es garantía de ausencia de síntomas ni de sitio proximal de bloqueo.

El substrato anatómico de los casos estudiados hasta ahora ha sido invariablemente una interrupción fibrosa del sistema éxcito-conductor atrioventricular, sea entre el nódulo y la muscu- latura auricular, en cuyo caso el nódulo puede estar hipoplásico o incluso faltar, sea en el haz de $\mathrm{His}^{4}, 5$. Para la interpretación morfogenética de esta lesión ha sido necesario investigar el desarrollo normal del sistema éxcito-conductor atrioventricular, habiéndose formulado dos concepciones: que el nódulo, haz y ramas se forman unitariamente a partir de una sola estructura, que se extiende y ramifica progresivamente ${ }^{6}$. En este caso toda discontinuidad será secundarja, es decir se habrá producido después de formadas estas estructuras; sin embargo, la mayoría de los autores $y$ en especial las investigaciones recientes 7.6 .8 apoyan la idea de que estas estructuras tienen origen en distintos componentes que se unen posteriormente (Fig. 5 a y b). En tal caso se plantea la posibilidad de que algunas discontinuj. dades sean primarias, es decir, representen tejido fibroso que impidió la unión ulterior de los componentes ${ }^{9}$. Hoy parece demostrada la hipótesis sustentada entre otros por Aschoff ${ }^{10}$, Zahn ${ }^{11,12}$, Doerr $^{13}$ y más recientemente por Anderson $y$ cols. ${ }^{7}$ y Davies $y \cdot c o l s,{ }^{14}$ de que el nódu lo $\mathrm{A}-\mathrm{V}$ se forma a partir de dos componentes: uno proximal aportado por la musculatura auricular y en especial del seno coronario, y otro distal proveniente del primitivo anillo muscular atrioventricular (Fig. 5 a y b). Que el nódulo $\mathrm{A}-\mathrm{V}$ tenga un componente del seno coronario parece estar demostrando indirectamente con el hallazgo de van Mierop y cols. ${ }^{15}$ de dos nódulos sinusales en algunos casos de sime tría bilateral de las cavas superiores en el sindrome de Ivemark. Los bloqueos proximales representarian así discontinujdades primarias ${ }^{4,5}$ entre los dos com. ponentes nodales. Por otra parte las investigaciones de Anderson $y$ cols. ${ }^{7}$ indican que los fascículos persistentes del anil]o $\mathrm{A}-\mathrm{V}$ dan origen tanto a la parte distal, compacta, del nódulo como a la porción proximal, perforante, del haz de His, porción que se uniría con la parte distal del haz, originada, según Wenink ${ }^{\theta}$, del anillo bulboventricular o según Davies y cols. ${ }^{14}$ de las fibras internas del tabique ventricular. Según esto, sólo entre las dos porciones del haz se plantearia la posibilidad de otra discontinuidad primaria.

E] caso estudiado revela dos particularidades: por una parte presenta dos interrupciones del haz, por otra, marcados signos degenerativos tanto de la musculatura del haz como del tejido fibroso circundante con calcificaciones de tipo đistrófico. A pesar de que las investigaciones de uno de nosotros apoyan la idea de un origen no unitario del SEC atrioventricular, los hallazgos en este caso difícilmente pueden interpretarse como discontinujdades primarias: habría que aceptar 

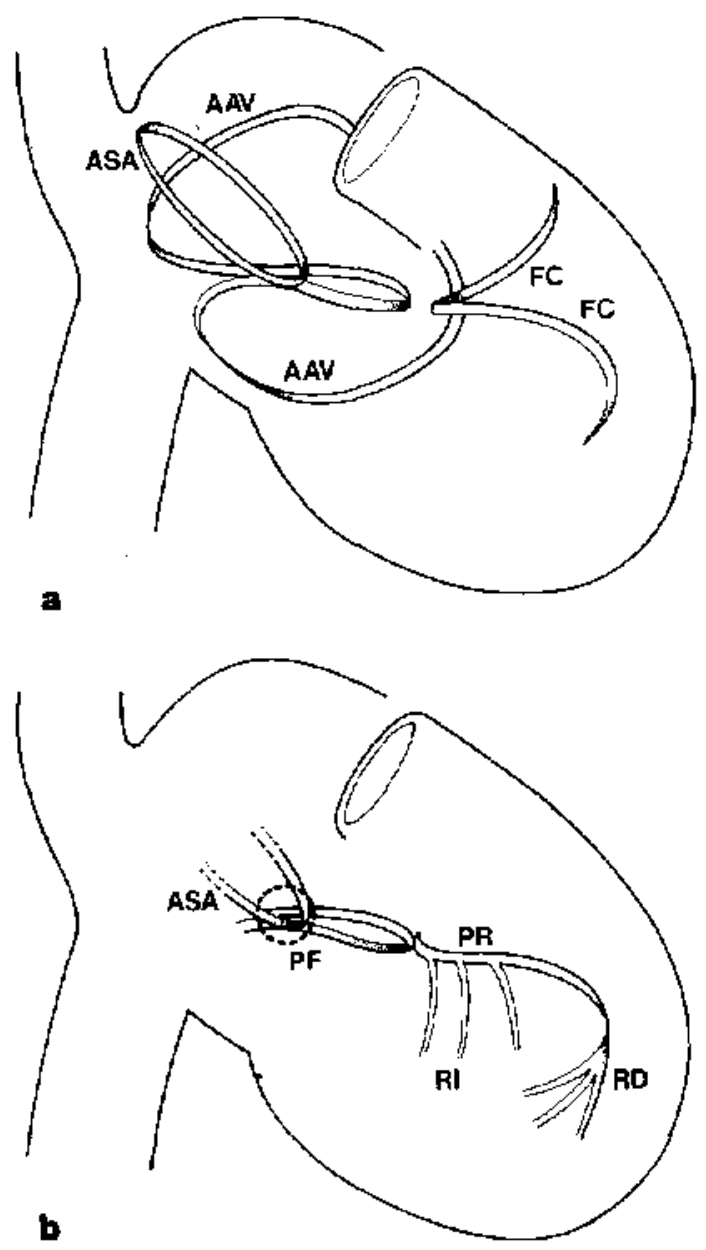

Fig. 5. Esquemas del corazón embrionario, visto desde la derecha, a) con las fibras internas primitivas que darían origen al sistema éxcito-conductor. ASA: anillo senoauricular, AAV: anillo atrioventricular, FC: fibras de contorno bulboventriculares. (Según Wenjnk ${ }^{8}$, modificado). b) con los segmentos principales del sistema éxcito-conductor atrioventricular. En la formación del nódulo atrioventricular (zona indicada por el círculo) participaria un componente del anillo senoauricular (ASA) y uno del anillo atrioventricular, de este último 3e desarrollaría también la porción perforante (PF) del haz. La porción ramificada del haz (PR) se originar fa de las fibras bulboventriculares (vease texto). Los sitios de las interrupciones primarias (por falta de unión de los componentes) estarían en el nódulo atrioventricular (zona del círculo) y entre las dos porciones indicadas del haz (PF y PR). RJ: ramas izquierdas, $\mathbf{R D}$ : rama derecha. (según Werink [8], modificado).

uñ tercer sitio de unión, a saber entre la porción compacta del nódulo y la perforante del haz. Por otro lado, los signos distróficos del tejido fibroso indican mas bien un proceso degenerativo que afectó secundariamente a estas 'estructuras. Davies y Anderson ${ }^{15}$ también se pronuncian en favor de esta interpretación para los casos de interrupción de la porción proximal del haz de
His. Es posible que la hipoxia neonatal haya causado una necrosis electiva del parénquima éxcito conductor de manera similar a la necrosis del miocardio de trabajo encontrada en el cuadro de la isquemia neonatal del miocardio.

Según la bibliografía revisada, este es el primer caso comunicado en que se encuentran dos interrupciones del haz con un segmento "desparenquimatizado" y en que se plantea razonablemente la hipótesis de que una hipoxia neonatal pueda ser la causa de un bloqueo $\mathrm{A}-\mathrm{V}$.

\section{RESUMEN}

Se presenta el caso de un bloqueo A-V congénito aislado en una niña de cuatro meses de edad, nacida de parto prematuro con asfixia neonatal. El examen anátomo-patológico demostró: nódulo A-V bien conformado, dos interrupciones fibrosas del haz de $\mathrm{His:}$ una en el segmento perforante y la otra en el segmento ramificado; entre ambas, un segmento de haz de His sin fjbras musculares específicas y sólo con tejido conjuntivo, en el tejido fibroso circundante, algunos depósitos calcáreos. Se discute la patogenia de las lesiones encontradas en relación con las jdeas actuales sobre el desarrollo del sistema excito-conductor del hombre. Se con. cluye que las lesiones descritas no corresponden a las descritas en los casos de bloqueo por trastornos del desarrollo embrionario, y que probablemente ellas con el resultado de un proceso degenerativo que afectó secundariamente a estas estructuras. Se considera la posibilidad de que dicho proceso haya sido la hipoxia neonatal con consecuente necrosis electiva del parénquima específico. Según la bibliografia revisada, el caso comunicado es el primero con dos interrupciones del sistema $\mathrm{A}-\mathrm{V}$.

\section{REFERENCIAS}

1. Roberts, N., Gelband, H.: Arrythmias: in Moss: A.J., Adans, F.H, and Emmanouillides, G.C.: Heart disease in infants, children and adolescents. Edition 2. Baltimore, williams and Wikins Co., 1977.

2. Michaelsson, $\boldsymbol{M}$, and Engle, M.A.: Congenital Complete Heart Block: An international study of the natural history. Cardiovsc. Clin. 4: 86, 1972.

3. Esscher, E.: Congerital complete heart block. A clinical study. Upprala. Acta Universitatis Upsaliensis. 1979.

4. Anderson, R.H., Wenick, A.C.G., Losekoot. T.G., and Becker, A.E.: Congenitally Complete Heart Block. Developmental Aspects. Circulation 56: 90 , 1977.

5. Lev, $M$ : Pathogenests of Congenital Atrioventricular Block. Progr. Cardiovasc. Dis. 15: 145, 1972.

6. Chuaqui, B.: Zur Histogenese des A $-V$ Knotens bein Mensthen. Basic Res. Cardjol. 6B: 266, 1973. 
7. Anderson, R.H., Becker, A.E., and Wenink, C.G.: The Development of the Conducting tissues. Lin: Cardiac Arrythmias in the Neonate, lnfant, and Child.; ed. N.K. Roberts and H. Getband. New York: Appleton-Century-Crofts 1977b, pag. $1-28$.

8. Wenink, A.C.G.: Deyelopment of the Human Cardiac Conducting System. J. Anat. $121: 617$, 1976.

9. James, Th. N.: Cardiac Conduction System: Fetal and Postnatal Development. Amer. J. Cardiol. 25: 213,1970 .

10. Aschoff, $L_{\text {.: }}$ Referat über die Herzstörungen in iheren Beziehungen zu dem spexifjschen Muskelsystems des Herzens. Verh. Dtsch, path. Ges. 14: 3, 1910.

11. Zahn, A.: Fxperimentelle Untersuchungen über Reizbildung in Atrioventrikularknoten und Sints coronarius. Zbt. Physiol. 26: 495, $1913 \mathrm{a}$.

12. Zahn, A.: Experimentelle Untersuchungen über Reizbilung und Reizleitung in Atrioventrikularknoten. Pfluger's Arch. ges. Physiol. 151: 247, $1913 \mathrm{~b}$.

13. Doerr. W.: Aligemeine Pathologie der Organe des Kreislaufe. En: Handbuch der allgemeinen Pathologie. Regidiert von H. Meesen, und F. Roulet. Bd. III/4. Springer: Berlin-Heidelberg-New York 1970, pág. 205 .

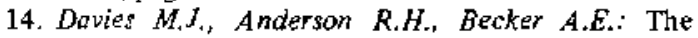
conduction system of the heart. Butterworths, London Boston Durban Singapore Sydney Toronto Wellington, 1983.

15. Von Nierop, L.H.S., Patterson, P.R., Reynolds, R.W.: Two Cases of Congenital asplenia with isomerism of the cardiac atria and sinoatrial nodes. Amer. J. Cardiol. 13: 407, 1964. 